\section{Fatores associados à ideação suicida na comunidade: um estudo de caso-controle}

\author{
Factors associated with suicidal ideation \\ in the community: a case-control study
}

\author{
1 Faculdade de Ciências \\ Médicas, Universidade \\ Estadual de Campinas, \\ Campinas, Brasil. \\ Correspondência \\ V. F. Silva \\ Departamento de Psicologia \\ Médica e Psiquiatria \\ Faculdade de Ciências \\ Médicas, Universidade \\ Estadual de Campinas. \\ Cidade Universitária \\ Zeferino Vaz, C. P. 6111 \\ Campinas, $S P$ \\ 13081-970, Brasil. \\ vivi.franco@bol.com.br
}

\begin{abstract}
This paper aims to identify variables associated with suicidal ideation during the previous 12 months in the city of Campinas, São Paulo, Brazil. Using a case-control design, risk factors for suicidal ideation were investigated through analysis of independent variables related to the individual, family, and health. Some 29 cases of suicidal ideation and 166 controls were interviewed. Cases were identified through a previous cross-sectional study, and controls were randomly selected from the same population base among those with no history of suicidal ideation in the previous 12 months. Regression models were used to control for confounders and effect modifiers. According to the results, demographic variables were not associated with suicidal ideation. In the final model, the variables that remained statistically significant were depressive symptoms, emotional difficulties, lack of neighborhood support, and less frequent church attendance. Suicidal ideation was consistently associated with factors related to depressive symptoms, especially lack of energy and depressed mood.
\end{abstract}

Suicide; Mental Health; Case-control Studies
Viviane Franco da Silva 1 Helenice Bosco de Oliveira 1 Neury José Botega 1 Letícia Marín-León 1 Marilisa Berti de Azevedo Barros 1 Paulo Dalgalarrondo 1

\section{Introdução}

No intuito de desenvolver métodos para o estudo do comportamento suicida em diferentes contextos, avaliar estratégias de tratamento e estabelecimento de bases científicas para futuras pesquisas, lançou-se o Estudo Multicêntrico de Intervenção no Comportamento Suicida (SUPRE-MISS), da Organização Mundial da Saúde (OMS) 1 . Trata-se de um projeto transcultural realizado em oito países (Brasil, Estônia, Índia, Irã, China, África do Sul, Sri Lanka e Vietnam) dentro de uma iniciativa destinada à prevenção do suicídio, com a supervisão científica do Australian Institute for Suicide Research and Prevention, Griffith University (Brisbane, Austrália) e do National Centre for Suicide Research and Prevention of Mental Ill-Health, Karolinska Institute (Estocolmo, Suécia).

Na cidade de Campinas, São Paulo, Brasil, foi realizado em 2003 um inquérito de base populacional, constituinte do projeto SUPRE-MISS que revelou os casos de ideação suicida, focalizados neste trabalho 2 .

Alguns estudos associam ideação suicida ao risco de tentativas de suicídio ${ }^{3}$ e estima-se que $60 \%$ dos indivíduos que se suicidam tinham, previamente, ideação suicida 4 . A gravidade e a duração dos pensamentos suicidas correlacionam-se com a probabilidade de tentativa de suicídio, que é, por sua vez, o principal fator de risco para suicídio completo 5. Evi- 
denciam-se, então, relações importantes entre pensamento e ato no contexto dos comportamentos suicidas, ainda que outros autores defendam tendências à não progressão da ideação para outras etapas do comportamento suicida 6 .

Dois estudos epidemiológicos recentes também demonstraram associação entre ideação e tentativas de suicídio. Pirkis et al. 7, na Austrália, investigaram uma amostra de 10.641 sujeitos, $16 \%$ dos quais tinham tido ideação suicida ao longo da vida. Nesse subgrupo, $12 \%$ tentaram suicídio num período de um ano de seguimento. Kuo et al. 8, em Baltimore (Estados Unidos), no intuito de compreender aspectos relacionados ao surgimento da ideação, realizaram um estudo de corte prospectivo de 3.481 sujeitos, encontrando uma incidência de $10 \%$ de tentativas de suicídio em pessoas que, na entrevista de base, 13 anos antes, relataram ideação suicida comparada com 1,6\% de incidência de tentativas para pessoas que não relataram ideação.

Pacientes com ideação suicida são usuários freqüentes de serviços ambulatoriais e de internações em saúde geral e mental com maior demanda para aconselhamento, informações e uso de medicação, freqüentemente insatisfeitos quanto ao atendimento de suas necessidades 9 . Há evidências de que intervenções específicas, realizadas em serviços de atenção primária, são efetivas em reduzir ou resolver ideação suicida e de que são freqüentes os atendimentos clínicos nos meses precedentes a uma tentativa 10 .

Além disso, grande parte do trabalho de prevenção do suicídio fundamenta-se na identificação de fatores de risco, seja através da elaboração de estratégias de prevenção específicas para grupos específicos, ou estratégias de base populacional. Uma caracterização atenta da parcela da população que manifesta ideação suicida pode revelar especificidades que refinem o potencial de diagnosticar e agir em tempo hábil.

Pesquisas referentes à ideação suicida e tentativas de suicídio geralmente utilizam amostras clínicas. São menos freqüentes as pesquisas na população geral 11, e o planejamento de saúde pública sabidamente requer dados populacionais. Estudo recente, de base populacional, realizado no Brasil, mostrou prevalência de $17,1 \%$ para ideação suicida ao longo da vida e 5,3\% para ideação no último ano ${ }^{2}$. Com a relativa escassez de estudos na comunidade, especialmente em países em desenvolvimento, que apontem para a questão, é proposto como objetivo deste estudo identificar variáveis as- sociadas à ideação suicida nos últimos 12 meses, na cidade de Campinas.

\section{Material e método}

Estuda-se aqui um subgrupo de 29 indivíduos dentre os 515 entrevistados durante o SUPREMISS, que responderam "sim" às duas questões consecutivas relacionadas à ideação suicida: (1) "Alguma vez você já pensou em pôr fim a sua vida?”; (2) "Este pensamento lhe ocorreu alguma vez nos últimos 12 meses?”.

O delineamento tipo caso-controle incluiu 29 casos de ideação e os 166 controles, selecionados aleatoriamente entre os que não apresentaram pensamento suicida nos últimos 12 meses. Isso perfaz a razão de um caso para 5,7 controles. Considerou-se um poder estatístico de $80 \%$ para detectar uma razão de chances de 3,6 com um nível de significância de 5\%.

Partiu-se da hipótese que os indivíduos com ideação no último ano apresentam caracterização diversa do grupo sem ideação, com maiores índices de morbidade psiquiátrica, incluindo uso de drogas e álcool, mais problemas físicos e utilização mais freqüente dos serviços de saúde.

Os casos e controles foram entrevistados através de questionário padronizado, com a versão final elaborada pela equipe de pesquisadores principais do SUPRE-MISS 1. As questões foram originalmente desenvolvidas em inglês. Realizou-se um estudo piloto onde foi avaliada a equivalência semântica. $O$ instrumento permitiu obter informações sócio-demográficas, história de comportamento suicida pessoal e em membros da família, saúde física e mental, contato com serviços de saúde e questões relacionadas com consumo de álcool e drogas.

Duas escalas psicométricas foram acrescentadas ao protocolo local: o Self-Report Questionnaire (SRQ-20) 12 e o Alcohol Use Disorder Identification Test (AUDIT) 13, já validados no Brasil. O AUDIT identifica usuários de álcool que se encontram numa faixa de risco; pontuação igual ou superior a oito (variação 0 a 40) é sugestiva de uso abusivo e/ou dependência do álcool. O SRQ-20 contém vinte questões com respostas sim/não que rastreiam morbidade psiquiátrica não-psicótica; pontuações iguais ou superiores a oito, numa escala de 0 a 20 , foram utilizadas como indicativas de morbidade psiquiátrica.

A partir da estrutura fatorial do SRQ-20 proposta por Iacoponi \& Mari 14, foram criadas quatro novas variáveis: "falta de energia" (questões do SRQ-20 de números: 8, 11, 12, 13, 18 e 
20), "sintomas somáticos" (questões do SRQ-20 de números: 1, 2, 7 e 19), "estado depressivo" (questões do SRQ-20 de números: 6, 9 e 10) e "pensamentos depressivos" (questões do SRQ20 de números: 14, 15, 16 e 17).

As questões relacionadas ao uso de álcool e drogas também foram avaliadas através de resposta às seguintes questões:

- Alguma vez na vida, você já experimentou alguma das seguintes substâncias?

a) Produtos de tabaco (cigarro, cachimbo, charuto etc.) [não/sim];

b) Bebidas alcoólicas (cerveja, vinho, licor etc.) [não/sim];

c) Maconha [não/sim];

d) Cocaína ou crack [não/sim];

e) Estimulantes ou anfetaminas (pílulas dietéticas, ecstasy etc.) [não/sim];

f) Inalantes (solventes, colas, thinner) [não/ sim];

g) Calmantes ou pílulas soníferas (Valium, Lorax, Dormonid etc.) [não/sim];

h) Alucinógenos (LSD, chá de cogumelo etc.) [não/sim];

i) Heroína, morfina, metadona ou analgésicos opióides etc. [não/sim];

j) Outros [especificar].

- Se "sim" em algum desses itens, nos últimos 3 (três) meses, com que freqüência você usou as substâncias que mencionou? [nunca/uma ou duas vezes/uma vez por mês/uma vez por semana/diariamente ou quase].

As "dificuldades emocionais" relatadas foram caracterizadas a partir da questão: "Você já teve ou tem tido dificuldades emocionais, por longos períodos de tempo [mínimo de um ano] que o impediram de realizar bem as tarefas do dia-a-dia?".

A avaliação da solidariedade de vizinhos foi feita através da questão: "Em sua opinião, quão próximas umas das outras estão as pessoas $e$ quanto elas se ajudam?", para valores de 1 (distantes/não se ajudam) a 5 (muito próximas/se ajudam muito). O ponto de corte considerado para essa variável foi 3.

Quanto à variável "religião", observamos que se trata da filiação religiosa, em que o entrevistado respondeu pela denominação de sua religião. Já a variável "freqüência à igreja” foi avaliada com a seguinte questão: "Com que freqüência você vai à igreja [ou outro lugar de culto]?" (uma vez por semana/uma vez por mês/ duas a três vezes por ano/aproximadamente uma vez por ano/quase nunca).

Tratamento psiquiátrico anterior foi avaliado mediante dois grupos de questões referentes à história de internações psiquiátricas e tratamento psiquiátrico ambulatorial. Respostas afirmativas a pelo menos uma das questões eram requeridas para se considerar tratamento psiquiátrico anterior. As seguintes questões foram utilizadas:

- História de internações psiquiátricas: Você já precisou de internação por problemas mentais? [sim/não].

- Tratamento psiquiátrico ambulatorial: Você teve contato com algum dos seguintes serviços profissionais para tratamento psiquiátrico?

a) Serviços psiquiátricos, posto ou ambulatório [não/sim];

b) Serviços psiquiátricos, CAPS [não/sim];

c) Internação parcial (hospital-dia) [não/sim];

d) Psicólogos e psiquiatras particulares [não/ sim];

e) Serviços de consultório para problemas relacionados ao álcool e drogas [não/sim].

Quanto às variáveis sócio-demográficas, convém observar que a variável "renda" referese à renda anual, individual. Como estudo multicêntrico, realizado em diversos países, o banco de dados do SUPRE-MISS, no que tange à renda, foi descrito em dólares. Decidimos mantê-lo em virtude da maior estabilidade da moeda.

A existência de associação entre ideação suicida e cada um dos fatores foi verificada considerando as variáveis constantes nas tabelas. A análise dos dados foi realizada no programa Stata versão 7.0 (Stata Corporation, College Station, Estados Unidos). Inicialmente procedeuse à análise univariada de todas as informações coletadas, com cálculo das proporções para as variáveis categóricas. Em seguida foram realizadas as análises bivariadas, observando-se as proporções das exposições entre casos e controles, os testes de qui-quadrado e as respectivas odds ratios (OR) e seus intervalos de confiança de 95\% (IC95\%). Na etapa seguinte, procedeu-se à análise multivariada, mediante regressão logística não-condicional, considerando o delineamento não-pareado dos casos e controles. O processo foi iniciado pelo modelo saturado com as variáveis com valor de p menor que $20 \%$ e com eliminação posterior daquelas com p maior ou igual a $5 \%$.

\section{Resultados}

A Tabela 1 apresenta os resultados referentes ao efeito isolado das variáveis sócio-econômicas, sendo que apenas renda entre 1 e 2.500 dólares mostrou-se associada à ideação, mas com valor de p de 10\%. Quanto à variável "escolaridade", verificou-se uma diminuição da chance de pensamento suicida com o aumento do número de anos cursados, mas sem significância 
Características sócio-demográficas e religiosas dos casos e controles.

\begin{tabular}{|c|c|c|c|c|c|c|c|}
\hline \multirow[t]{2}{*}{ Características } & \multicolumn{2}{|c|}{ Casos ( $N=29)$} & \multicolumn{2}{|c|}{ Controles $(N=166)$} & \multirow[t]{2}{*}{ OR } & \multirow[t]{2}{*}{ IC95\% } & \multirow[t]{2}{*}{ Valor $p$} \\
\hline & $\mathrm{n}$ & $\%$ & $\mathrm{n}$ & $\%$ & & & \\
\hline \multicolumn{8}{|l|}{ Gênero } \\
\hline Masculino & 12 & 41,4 & 62 & 37,3 & 1,00 & $0,49-2,83$ & 0,84 \\
\hline Feminino & 17 & 58,6 & 104 & 62,7 & 1,18 & & \\
\hline \multicolumn{8}{|l|}{ Faixa etária (anos) } \\
\hline $14-39$ & 13 & 44,8 & 91 & 54,8 & 1,07 & $0,29-4,24$ & 0,99 \\
\hline $40-59$ & 12 & 41,4 & 45 & 27,1 & 2,00 & $0,53-8,19$ & 0,40 \\
\hline $60 e+$ & 4 & 13,8 & 30 & 18,1 & 1,00 & & \\
\hline \multicolumn{8}{|l|}{ Escolaridade (anos) } \\
\hline $0-8$ & 2 & 6,9 & 23 & 13,8 & 1,00 & & \\
\hline $9-11$ & 18 & 62,1 & 71 & 42,8 & 2,92 & $0,58-19,69$ & 0,24 \\
\hline 12 e + & 9 & 31,0 & 72 & 43,4 & 1,44 & $0,26-10,42$ & 0,99 \\
\hline \multicolumn{8}{|l|}{ Renda (US\$)* } \\
\hline Sem renda & 10 & 34,5 & 42 & 25,3 & 1,00 & & \\
\hline $1-2.500$ & 5 & 17,2 & 61 & 36,8 & 0,34 & $0,09-1,20$ & 0,11 \\
\hline $2.501-10.000$ & 11 & 37,9 & 47 & 28,3 & 0,98 & $0,34-2,82$ & 0,84 \\
\hline $10.001 e+$ & 3 & 10,4 & 16 & 9,6 & 0,79 & $0,15-3,73$ & 0,99 \\
\hline \multicolumn{8}{|l|}{ Situação conjugal } \\
\hline Solteiro & 7 & 24,2 & 53 & 31,9 & 1,00 & & \\
\hline Casado/Amasiado & 17 & 58,6 & 84 & 50,6 & 1,53 & $0,55-4,40$ & 0,51 \\
\hline Viúvo/Divorciado & 5 & 17,2 & 29 & 17,5 & 1,31 & $0,32-5,16$ & 0,75 \\
\hline \multicolumn{8}{|l|}{ Religião (N = 179) } \\
\hline Católica & 19 & 70,4 & 110 & 72,4 & 1,00 & & \\
\hline Outras & 8 & 29,6 & 42 & 27,6 & 1,10 & $0,41-2,92$ & 0,98 \\
\hline \multicolumn{8}{|l|}{ Freqüência à igreja } \\
\hline Menos que uma vez/mês & 13 & 48,2 & 44 & 28,8 & 2,30 & $0,93-5,71$ & 0,08 \\
\hline Pelo menos uma vez/mês & 14 & 51,8 & 109 & 71,2 & & & \\
\hline
\end{tabular}

* Em julho de 2003, período do encerramento das entrevistas, a cotação do Dólar era de $\mathrm{R} \$ 2,86$.

estatística. Odds ratio para 9-11 anos de estudo foi 2,92 (IC95\%: 0,58-19,69) e para 12 anos e mais 1,44 (IC95\%: 0,26-10,42), comparados com a categoria até oito anos.

Para as variáveis relativas à situação empregatícia, viver só ou com outras pessoas, separações conjugais, ter filhos, ser responsável por filho menor que 16 anos, também não se obteve evidência de associação estatisticamente significante no nível de $5 \%$.

A Tabela 2 evidencia que variáveis relacionadas à saúde mental e de comportamento relacionado à saúde estiveram associadas à ideação suicida. Indivíduos qualificados como positivos pelo SRQ-20 apresentaram quatro vezes mais chance para ideação que seus controles.
Indivíduos que referiram falta de energia, humor depressivo e pensamento depressivo tiveram mais chance para ideação $(\mathrm{OR}=4,78 ; 4,38$ e 4,99 respectivamente).

A avaliação da vizinhança no quanto é solidária, no quanto as pessoas se ajudam, apresentou-se significativa. Odds ratio para ideação foi de 3,9 (IC95\%: 1,33-12,31) quando esta vizinhança era sentida como distante (Tabela 2).

A presença de doenças ou problemas físicos nos últimos 12 meses e o uso de drogas ilícitas não estiveram associados à ideação, na análise univariada, diferentemente do uso de calmantes (Tabela 2).

Na Tabela 3 é apresentado o resultado final a partir da utilização da análise multivariada. A 
Tabela 2

Características de saúde mental e de comportamento relacionado à saúde entre casos e controles.

\begin{tabular}{|c|c|c|c|c|c|c|c|}
\hline \multirow[t]{2}{*}{ Exposição } & \multicolumn{2}{|c|}{ Casos } & \multicolumn{2}{|c|}{ Controles } & \multirow[t]{2}{*}{ OR } & \multirow[t]{2}{*}{ IC95\% } & \multirow[t]{2}{*}{ Valor $\mathrm{p}$} \\
\hline & $n$ & $\%$ & $\mathrm{n}$ & $\%$ & & & \\
\hline \multicolumn{8}{|c|}{ SRQ-20 (caso presumível) } \\
\hline Positivo & 15 & 51,7 & 34 & 20,5 & 4,16 & $1,70-10,22$ & $<0,01$ \\
\hline Negativo & 14 & 48,3 & 132 & 79,5 & & & \\
\hline \multicolumn{8}{|c|}{ SRQ-20 (falta de energia) } \\
\hline Sim & 16 & 55,2 & 34 & 20,5 & 4,78 & $1,95-11,80$ & $<0,01$ \\
\hline Não & 13 & 44,8 & 132 & 79,5 & & & \\
\hline \multicolumn{8}{|c|}{ SRQ-20 (sintomas somáticos) } \\
\hline Sim & 18 & 62,1 & 80 & 48,2 & 1,76 & $0,73-4,27$ & 0,24 \\
\hline Não & 11 & 37,9 & 86 & 51,8 & & & \\
\hline \multicolumn{8}{|c|}{ SRQ-20 (humor depressivo) } \\
\hline Sim & 13 & 44,8 & 26 & 15,7 & 4,38 & $1,74-11,03$ & $<0,01$ \\
\hline Não & 16 & 55,2 & 140 & 84,3 & & & \\
\hline \multicolumn{8}{|c|}{ SRQ-20 (pensamento depressivo) } \\
\hline $\operatorname{Sim}$ & 18 & 62,1 & 41 & 24,7 & 4,99 & $2,03-12,42$ & $<0,01$ \\
\hline Não & 11 & 37,9 & 125 & 75,3 & & & \\
\hline \multicolumn{8}{|c|}{ AUDIT (caso presumível) } \\
\hline Positivo & 4 & 13,8 & 14 & 8,4 & 1,74 & $0,44-6,31$ & 0,31 \\
\hline Negativo & 25 & 86,2 & 152 & 91,6 & & & \\
\hline \multicolumn{8}{|c|}{ Tratamento psiquiátrico anterior } \\
\hline Sim & 9 & 31,0 & 32 & 19,3 & 1,88 & $0,72-4,88$ & 0,24 \\
\hline Não & 20 & 69,0 & 134 & 80,7 & & & \\
\hline \multicolumn{8}{|c|}{ Dificuldades emocionais relatadas } \\
\hline Sim & 18 & 62,1 & 38 & 22,9 & 5,51 & $2,23-13,79$ & $<0,01$ \\
\hline Não & 11 & 37,9 & 128 & 77,1 & & & \\
\hline \multicolumn{8}{|c|}{ Tentativa suicídio na vida } \\
\hline Sim & 5 & 17,2 & 6 & 3,6 & 5,56 & $1,34-22,82$ & 0,01 \\
\hline Não & 24 & 82,8 & 160 & 96,4 & & & \\
\hline \multicolumn{8}{|c|}{ Uso de calmantes nos últimos três meses } \\
\hline Sim & 9 & 31,0 & 23 & 13,9 & 2,80 & $1,03-7,49$ & 0,03 \\
\hline Não & 20 & 69,0 & 143 & 86,1 & & & \\
\hline \multicolumn{8}{|c|}{ Avaliação da vizinhança $(N=194)$} \\
\hline Distante & 24 & 82,8 & 91 & 55,2 & 3,90 & $1,33-12,31$ & $<0,01$ \\
\hline Próxima & 5 & 17,2 & 74 & 44,8 & & & \\
\hline
\end{tabular}


Tabela 3

Resultado final com modelo de regressão logística não-condicional:

casos de ideação suicida no último ano e controles.

\begin{tabular}{lccc}
\hline Variáveis & OR & IC95\% & Valor $\mathbf{p}$ \\
\hline Humor depressivo & 3,80 & $1,325-10,925$ & 0,01 \\
Vizinhança não solidária & 3,57 & $1,134-11,240$ & 0,03 \\
Falta de energia & 3,22 & $1,216-8,540$ & 0,02 \\
Dificuldades emocionais relatadas & 2,65 & $1,011-6,930$ & 0,05 \\
Menor freqüência à igreja & 2,79 & $1,023-7,619$ & 0,05 \\
\hline
\end{tabular}

análise bruta sugeriu chance aumentada de ideação para uso de calmantes, porém, não foi mantida a significância estatística quando se fez o ajuste simultâneo para as outras variáveis.

\section{Discussão}

Em relação ao comportamento suicida, a maioria dos estudos enfatiza a questão das tentativas e do êxito letal. Para esses desfechos, numerosos fatores de risco já foram identificados. Quanto à ideação suicida, relativamente poucos estudos caracterizam os fatores associados. O presente estudo, pesquisando indivíduos na comunidade, sem os vieses representados por amostras de pacientes ambulatoriais ou hospitalizados, avaliou ideação suicida nos últimos 12 meses.

A utilização de estudos caso-controle, pela sua eficiência, é tipicamente empregada para doenças ou desfechos raros, numa abordagem em que diferenças entre os grupos, sendo esses comparáveis, são prováveis fatores de risco para a condição em estudo. Neste estudo, a análise multivariada permitiu achados significativos, principalmente quanto às variáveis relacionadas a sintomas depressivos, sugerindo que um relato de ideação suicida nos últimos 12 meses associa-se de forma consistente a esses sintomas.

Alguns estudos sugerem que os mesmos fatores de risco tendem a operar nas diferentes etapas do processo de suicídio 6 . Entre esses níveis, a ideação suicida pode ser o alvo de um dos níveis mais precoces passíveis de intervenção. O presente estudo busca especificidades da população que relata ideação suicida nos últimos 12 meses, mas observa-se, contudo, que existem limitações impedindo a afirmação de que as diferenças encontradas entre os grupos constituam fatores de risco. Tratando-se de um estudo caso-controle inserido num estudo seccional, abordam-se os achados deste estudo como possíveis fatores associados.

Se o conceito de suicídio, como ato, revelase complexo e repleto de divergências, pode-se pensar no quão instáveis e diversas são as definições correntes de ideação suicida. Primeiramente, as ideações a que se tem acesso são meramente as ideações "comunicadas". A limitação metodológica, então suscitada, é se há diferença entre um grupo que comunica e um grupo que não comunica ideação suicida. Acreditamos que são limitações inerentes a esse tipo de estudo já que "ideação suicida" (como pensamento) não se trata de uma variável passível de observação objetiva. Ao supor que os que responderam negativamente sobre a presença de ideação suicida, podem tê-lo feito por outras razões (valores morais, religiosos, empatia com o entrevistador etc.), admitimos a existência dessa possível fonte de viés: diferenças existentes podem não ser detectadas se o grupo controle estiver “contaminado” por ideação suicida não revelada. Contudo, procuramos enfatizar que a diferença a que nos propusemos a fazer seria entre o grupo que relata ideação e o grupo que não relata, ou seja, não a ideação como "pensamento", mas sim a ideação como "comunicação".

Dentre os 515 entrevistados, consideram-se então, neste estudo, os 29 que comunicaram, como os indivíduos que tiveram ideação suicida nos últimos 12 meses. Não se nega a existência de um contingente submerso, tanto no grupo de casos quanto no grupo-controle, que não se dispôs a revelar pensamentos a respeito de suicídio.

Os estudos, em geral, definem ideação suicida das mais variadas formas: relato espontâneo ou resposta a diferentes questionamentos, que abrangem desde pensamentos de que a vida não vale a pena ser vivida até preocupações intensas, quase delirantes 11.

Neste estudo, as questões utilizadas para identificar o grupo com ideação suicida nos últimos 12 meses, embora amenizem o discurso, não fazendo referência à palavra suicídio, não transpõem o desafio de verificar distinções entre idéias transitórias e idéias mais sólidas, persistentes, que talvez pudessem ser acessadas pelo uso de instrumentos específicos 15. Tratando-se de um inquérito comunitário, este estudo buscou simplificar a forma de abordagem, preterindo o uso de escalas a favor da formulação de questões mais diretas e simples, menos redundantes. Considerando a presença de ideação nos últimos 12 meses, e não na vida, espera-se reduzir possíveis vieses de memória. Contudo, o número de casos da 
amostra limita a interpretação de alguns resultados.

No presente estudo não foram observadas diferenças entre casos e controles quanto às variáveis sócio-demográficas. Estudos sobre ideação suicida na última semana não encontraram diferença quanto às variáveis sócio-demográficas, atribuindo vários fatores ao achado, como, por exemplo, o $n$ reduzido limitando o poder do estudo de encontrar associações estatisticamente significantes 6 ou apontando as diferenças sócio-demográficas como menos decisivas quando se trata de ideação, em comparação com outras manifestações do comportamento suicida (planejamento e tentativa de suicídio) 16.

Já estudos internacionais recentes apontam maior prevalência de ideação suicida associada a fatores sócio-demográficos como gênero feminino, ter uma condição conjugal tal como divorciado, separado ou solteiro 17. As mulheres, de modo geral, tendem a ter mais ideação suicida na vida 17 , mesmo havendo estudos que não encontram diferenças entre gêneros 18 . O estudo prospectivo de Kuo et al. 8, analisando os fatores sócio-demográficos, concluiu que apenas idade associa-se significativamente ao surgimento de ideação: mais jovens apresentam maior incidência. Apontam também a tendência para menor incidência de ideação entre afro-americanos e para mais casos entre indivíduos viúvos ou separados por ocasião da entrevista de base. Outros estudos mostram que ideação suicida pode ser mais prevalente entre indivíduos não casados e mais jovens 16 .

O presente estudo não encontrou associação entre a religião (filiação religiosa) e a incidência de ideação suicida. Quanto a considerar-se religioso também não houve diferença significativa entre os grupos, no entanto, menor freqüência à igreja (menos de uma vez por mês) esteve associada ao grupo que relatou ideação no último ano. Dados da literatura referem que ideação suicida incide sem diferenças significativas entre as diversas religiões 10 . Alguns estudos sugerem que a religiosidade, não se levando em consideração a afiliação religiosa, pode ter uma função protetora em relação ao suicídio. Em uma amostra de imigrantes latino-americanos, não foram encontradas relações entre religião e ideação suicida. Porém, a percepção da própria religiosidade e a freqüência à igreja foram negativamente associadas com ideação suicida 19. Infelizmente, no intuito de simplificar o questionário para facilitar a execução, optamos por abreviar a investigação de determinadas questões e com isso não pesquisamos a religião em que o indivíduo foi criado ou o tempo de conversão, que, certamente, propiciariam análises interessantes.

Quanto aos sintomas psiquiátricos avaliados, os achados deste estudo relacionados a humor depressivo e falta de energia, estão de acordo com os de Gili-Planas et al. 11 em que transtornos afetivos são a categoria mais freqüente de transtorno em sujeitos que apresentam ideação suicida. Também a diferença quanto às "dificuldades emocionais por mais de um ano", com a especificação de que "impedem a boa realização de tarefas do dia-a-dia”, pode ser atribuída aos sintomas depressivos e à falta de energia. A avaliação da vizinhança como distante ou não solidária, significativa para o grupo com ideação, pode relacionar-se a sentimentos de desesperança e solidão nesta população. Quando diferentes aspectos de sofrimento são avaliados, o sentimento de desesperança mostra-se fortemente associado à ideação suicida 6. Goldney et al. 20 apontam a importância do transtorno depressivo como fator contribuinte para ideação suicida na comunidade. Segundo estudos de autópsia psicológica, $60 \%$ dos suicídios transcorrem em indivíduos com sintomas depressivos significantes 21 . Estudos de risco atribuível na população observam que, se toda a depressão pudesse ser prevenida ou efetivamente tratada, $40-50 \%$ do comportamento suicida e da ideação suicida poderiam ser eliminados 7. Esta consistente associação entre quadros depressivos e ideação suicida afasta uma tendência de determinados autores a "normalizar" a ideação suicida, colocando a questão como algo intrínseco ao ser humano, pensar na existência e na possibilidade de voluntariamente pôr um fim a ela. Não há evidências que sustentem este ponto de vista.

Os achados quanto ao uso de álcool e outras drogas, diferentemente dos encontrados na literatura 6 , não permitem estabelecer diferenças entre os grupos quanto ao uso desses psicotrópicos. A literatura mostra que, para casos incidentes de ideação, por ocasião de uma entrevista de base, 13 anos antes, observa-se maior probabilidade de relatos de história de abuso ou dependência de drogas ou depressão 6 . No mesmo estudo, não houve associação entre diagnóstico de abuso ou dependência de álcool, transtorno do pânico, agorafobia, esquizofrenia, transtornos obsessivos compulsivos (TOC) e transtorno de personalidade anti-social e o surgimento de ideação suicida. O presente estudo não avaliou aspectos de comportamento relacionados a atuações anti-sociais, a não ser uso de álcool e outras drogas.

Se ideação suicida é seis vezes mais freqüente na população do que tentativas 22 e al- 
guns autores sugerem que não se pode ligar muito facilmente ideação suicida à tentativa efetiva de suicídio 22 ou defendem que há uma tendência à não progressão da ideação para outras etapas do comportamento suicida 6 , ainda assim, há evidências de que fatores como personalidade, controle de impulsos, suporte social e fatores culturais podem influenciar a seqüência ideação suicida - tentativa de suicídio - suicídio e, a despeito de algumas descontinuidades, as diferenças etiológicas que se estabelecem entre suicídio, tentativa de suicídio e ideação poderiam ser mais de grau do que de tipo 23. Fatores relacionados a estresse, suporte social, auto-estima, uso de álcool, de- pressão, desesperança e dor crônica parecem ser comuns às várias etapas do processo 6 .

Mesmo com o número reduzido da amostra, que limita a interpretação de alguns resultados, com possibilidade de não serem encontradas diferenças quando comparados os grupos, observou-se diferença significativa quanto às variáveis ligadas a sintomas depressivos. A presença de sintomas depressivos pode também alterar a percepção das relações sociais, proporcionando uma atitude mais pessimista frente à possibilidade de auxílio por vizinhos, ou mesmo pela igreja, e desamparo frente aos mais próximos.

\section{Resumo}

Este estudo tem como objetivo identificar variáveis associadas à ideação suicida ao longo dos últimos 12 meses, na cidade de Campinas, São Paulo, Brasil. Através de um estudo de caso-controle investigou-se fatores associados a ideação suicida mediante análise de variáveis independentes relacionadas ao indivíduo, à família e à saúde. Foram entrevistados 29 casos de ideação e 166 controles. Os casos foram identificados através de um inquérito de prevalência e os controles, selecionados aleatoriamente da mesma base populacional, entre os que não relataram pensamento suicida nos últimos 12 meses. Modelos de análise de regressão foram propostos para controlar a ação dos fatores de confusão ou modificadores de efeito. Nos resultados obtidos, as variáveis demográficas não estiveram associadas à ideação. Na análise final permaneceram com significância estatística as variáveis falta de energia e humor deprimido, derivadas do SRQ-20, dificuldades emocionais relatadas, vizinhança não solidária e menor freqüência à igreja. Ideação suicida mostrou-se consistentemente associada a fatores relacionados a sintomas depressivos, principalmente falta de energia e humor deprimido.

Suicídio; Saúde Mental; Estudos de Casos e Controles

\section{Colaboradores}

V. F. Silva participou no levantamento bibliográfico, redação da introdução e discussão do artigo. H. B. Oliveira colaborou com a realização das análises estatísticas e redação de material e método e resultados. N. J. Botega, L. Marin-León, M. B. A. Barros e P. Dalgalarrondo contribuíram na discussão dos resultados, com sugestões para a redação e revisões da redação do artigo. 


\section{Referências}

1. World Health Organization. Multisite intervention study on suicidal behaviours SUPRE-MISS: protocol of SUPRE-MISS. Geneva: World Health Organization; 2002.

2. Botega NJ, Barros MBA, Oliveira HB, Dalgalarrondo P, Marín-León L. Suicidal behavior in the community: Prevalence and factors associated with suicidal ideation. Rev Bras Psiquiatr 2005; 27:4553.

3. Kessler RC, Borges G, Walters EE. Prevalence of and risk factors for lifetime suicide attempts in the National Comorbidity Survey. Arch Gen Psychiatry 1999; 56:617-26.

4. Fawcett J, Clark DC, Bush KA. Assessing and treating the patient at risk for suicide. Psychiatr Ann 1993; 23:244-56.

5. Suominen K, Isometsä E, Suokas J, Haukka J, Achte K, Lönnqvist J. Completed suicide after a suicide attempt: a 37-year follow-up study. Am J Psychiatry 2004; 161:563-4.

6. Vilhjalmsson R, Kristjansdottir G, Sveinbjarnardottir E. Factors associated with suicide ideation in adults. Soc Psychiatry Psychiatr Epidemiol 1998; 33:97-103.

7. Pirkis J, Burgess P, Dunt D. Suicidal ideation and suicide attempts among australian adults. Crisis 2000; 21:16-25.

8. Kuo WH, Gallo JJ, Tien AY. Incidence of suicide ideation and attempts in adults: the 13-year follow-up of a community sample in Baltimore, Maryland. Psychol Med 2001; 31:1181-91.

9. Pirkis JE, Burgess PM, Meadows GN, Dunt DR. Suicidal ideation and suicide attempts as predictors of mental health service use. Med J Aust 2001; 175:542-5.

10. Bruce ML, Ten Have TR, Reynolds CF 3rd, Katz II, Schulberg HC, Mulsant BH, et al. Reducing suicidal ideation and depressive symptoms in depressed older primary care patients: a randomized controlled trial. JAMA 2004; 291:1081-91.

11. Gili-Planas M, Rocca-Bennasar M, Ferrer-Perez V, Bernardo-Arroyo M. Suicidal ideation, psychiatric disorder, and medical illness in a community epidemiological study. Suicide Life Threat Behav 2001; 31:207-13.

12. Mari JJ, Williams P. A validity study of a psychiatric screening questionnaire (SRQ-20) in primary care in the city of Sao Paulo. Br J Psychiatry 1986; 148:23-6.
13. Barbor TE, La Fuente JR, Saunders J, Grant M. AUDIT: the alcohol use disorders identification test: guidelines for use in primary health care. Geneva: World Health Organization; 1992.

14. Iacoponi E, Mari JJ. Reliability and factor structure of the portuguese version of self-reporting questionnaire. Int J Soc Psychiatry 1988; 35:213-22.

15. Beck AT, Kovacs M, Weissmann A. Assessment of suicide intention: the scale for suicide ideation. J Consult Clin Psychol 1979; 47:343-52.

16. Sorenson SB, Rutter CM Transgerational patterns of suicide attempt. J Consult Clin Psychol 1991; 59:861-6.

17. Weissman MM, Bland RC, Canino GJ, Greenwald S, Hwu HG, Joyce PR. Prevalence of suicide ideation and suicide attempts in nine countries. Psychol Med 1999; 29:9-17.

18. Renberg ES. Self-reported life-weariness, deathwishes, suicidal ideation, suicidal plans and suicide attempts in general population surveys in the north of Sweden 1986 and 1996. Soc Psychiatry Psychiatr Epidemiol 2001; 36:429-36.

19. Hovey JD. Religion and suicidal ideation in a sample of Latin American immigrants. Psychol Rep 1999; 85:171-7.

20. Goldney RD, Fisher LJ, Wilson DH, Dal Grande EF. Population attributable risk of major depression for suicidal ideation in a random and representative community sample. J Affect Disord 2003; 74:267-72.

21. Lonnqvist JK. Psychiatric aspects of suicidal behavior: depression. In: Hawton $\mathrm{K}$, van Heeringen $\mathrm{K}$, editors. The international handbook of suicide and attempt suicide. Chichester: Wiley; 2000. p. 107-20.

22. Bertolote JM, Fleischmann A, De Leo D, Bolhari J, Botega N, De Silva D, et al. Suicide attempts, plans, and ideation in culturally diverse sites: the WHO SUPRE-MISS community survey. Psychol Med 2005; 35:1457-65.

23. Gunnell D, Frankel S. Prevention of suicide: aspirations and evidence. BMJ 1994; 308:1227-33.

Recebido em 26/Jul/2005

Versão final reapresentada em 20/Dez/2005

Aprovado em 26/Dez/2005 